\title{
Enhancement of Germ Cell Apoptosis Induced by Chronic Ethanol in Transgenic Mice Overexpressing Fas Ligand \\ $\mathrm{C} \mathrm{Xu}^{1}, \mathrm{Z} \mathrm{Xiang}^{1}, \mathrm{D} \mathrm{Wu}^{1}, \mathrm{~J}_{\mathrm{Zhang}^{2}}$, W Le ${ }^{1}$
}

\begin{abstract}
Objective: To analysis ethanol exposure and its apoptosis results in germline cells.

Methods: Transgenic mouse model ubiquitously overexpressing human FasL was applied to investigate whether Fas ligand played a role in ethanol-induced testicular germ cell apoptosis. Wild-type (WT) and transgenic (TG) mice were treated with acute ethanol $(20 \% \mathrm{v} / \mathrm{v})$ by intraperitoneal injection five times. Flow cytometry, western blot and histopathological examination were carried out to estimate the apoptosis and FasL expression status in germline cells.

Results: Flow cytometry analysis showed that the percentage of FasL-positive cells was increased by $5.55 \%$ by ethanol while compared with control group (3.91\%). The increaesd expression of the FasL protein in ethanol treated cells analyzed by western blot was consistent with the result of Flow cytometry.

The apoptotic cell death was observed by PI staining of mouse testis tissue. Consequently, ethanol-treated WT mice displayed increased apoptotic cells while compared with saline-treated WT mice, besides, the FasL transgenetic mice displayed severe injury of spermatogonia and spermatocytes.
\end{abstract}

Conclusion: Chronic ethanol exposure can result in testicular germ cell apoptosis. Our present study provided direct evidence demonstrating that Fas ligand mediated apoptosis in testicular germ cells induced by acute ethanol using FasL transgenic mice.

Keywords: Apoptosis, ethanol, fas ligand, testes, transgenic mouse

From: ${ }^{1}$ Department of Urology, Tongji Hospital, Tongji University School of Medicine, 389 Xincun Road, Shanghai, 200065 China.

${ }^{2}$ Department of Urology, Tongren Hospital, Shanghai Jiaotong University School of Medicine, Shanghai, China.

Correspondence: Dr W Le, Department of Urology, Tongji Hospital, Tongji University School of Medicine, 389 Xincun Road, Shanghai, China. Fax: +86-021-64085875,

e-mail: lewei0411@163.com 


\section{INTRODUCTION}

Apoptosis in testicular germ cells is critical for spermatogenesis in mammals $(1,2)$. It is believed that apoptosis plays an important role in the removal of overproduced, genetically abnormal, or accidentally damaged germ cells. Furthermore, increases in germ cell apoptosis are observed in laboratory animals after exposure to various testicular toxicants $(3,4)$ and in humans after testicular injury or under certain disease conditions $(5,6)$.

Apoptosis is a rapid, self-destructive process that does not cause damage to surrounding tissues (7). Morphologically, the process of cell death is characterized by condensation and fragmentation of cell chromatin, followed by cellular budding into apoptotic bodies $(8,9)$. The mechanisms controlling spontaneous or toxicant-induced germ cell apoptosis are the current focus of many investigations. Recent studies have shown that the Fas-Fas ligand system is involved in the regulation of germ cell apoptosis both in rodent models (10) and human testes (11). Fas ligand (FasL/CD95L) is a $31-\mathrm{kDa}$ type II transmembrane protein of the tumor necrosis factor (TNF) family that induces apoptosis through interaction with Fas, a process best studied in lymphoid cells $(12,13)$. Fas (CD95/APO-1) is a type I transmembrane protein belonging to the family of TNF/nerve growth factor (NGF) receptors and has a broad distribution in vivo. In rodent testes, FasL is constitutively expressed in Sertoli cells, and Fas is located in selected germ cells (10).

Ethanol is among the most widely abused drugs in the world, and chronic alcohol abuse leads to testicular atrophy, feminization, and infertility in alcoholic men (14). Histological examination of these patients demonstrates a marked reduction in seminiferous tubular diameter accompanied with a loss of germ cells (14). The mechanisms of this injury were previously studied. In one mechanism, ethanol metabolism produces oxidative stress within the testes (15); alternatively, ethanol exposure changes either testosterone (16) or esterone levels (17). In addition, Zhu et al showed that ethanol exposure adversely affects the secretory 
function of Sertoli cells (18), and their recent research revealed enhanced apoptosis within the testes after chronic ethanol exposure (19). However, it is still not clear how ethanol can lead to testicular injury. Jankala et al demonstrated that the expression levels of FasL and p53 were enhanced after chronic ethanol exposure (20); however, no further evidence was provided. We have previously generated a model of transgenic mice ubiquitously expressing human FasL. Human FasL is known to show $76.7 \%$ amino acid sequence similarity with murine FasL. Furthermore, crossinteractions between human Fas/FasL and murine Fas/FasL induce apoptosis at the same efficiency (21). In this study, we used this FasL-overexpressing transgenic model to evaluate the functional importance of FasL in the regulation of germ cell apoptosis in the testes after chronic ethanol exposure.

\section{SUBJECTS AND METHODS}

\section{Animals}

FasL-overexpressing transgenic mice and wild-type littermates were got form Model Animal Research Center of Nanjing University. Adult mice (ages 3-4 months, matched sexes) were used for all experiments. TG, as used throughout this manuscript, refers to homozygotes with the FasL transgene (Tg2 in reference 20), whereas WT (wild-type) refers to littermate mice without the FasL transgene. Mice were housed in plastic mouse cages $(<4$ mice per cage) with free access to standard rodent chow and water. The colony room was maintained at $22.2^{\circ} \mathrm{C}$ with a 12-h:12-h light:dark cycle. WT and TG mice were fed $20 \%(\mathrm{v} / \mathrm{v})$ ethanol solution for 12 weeks, whereas control mice received the same volume of saline feeding. Mice were sacrificed, and the testes were removed after the final week of treatment. All the experimental protocols were performed in compliance with the National Institutes of Health guidelines for the care and use of animals and were approved by the local Animal Care and 
Use Committee.

\section{Flow cytometry analysis}

Single-cell suspensions of testicular cells were prepared by mechanical isolation and subsequently filtered through a hole density optimized nylon fiber. Samples were treated with $0.168 \mathrm{M} \mathrm{NH}_{4} \mathrm{Cl}$ to lyse erythrocytes and washed with $200 \mathrm{~mL}$ phosphate-buffered saline (PBS) twice. The cells were then blocked with $1 \%$ bovine serum albumin (BSA) in PBS for 20 min on ice, incubated with 1:100 rabbit anti-FasL antibodies (Santa Cruz Biotechnology, Santa Cruz, CA, USA) or rabbit serum as background control for $1 \mathrm{~h}$. Cells were then washed with PBS three times and subsequently exposed to fluorescein isothiocyanate (FITC)-conjugated anti-rabbit IgG goat polyclonal antibodies (Santa Cruz Biotechnology) in the dark for $1 \mathrm{~h}$. After three washes in PBS, samples were analyzed with a FACScan (Becton Dickinson, CA, USA) to determine the expression of FasL. Data were processed using a Hewlett Packard computer (Palo Alto, CA, USA). For propidium iodide (PI)-based flow cytometry, cells were stained with $100 \mathrm{~mL}$ of $50 \mathrm{mg} / \mathrm{mL}$ PI in $300 \mathrm{~mL}$ PBS ( $1 \mathrm{~h}$ on ice in the dark) and then measured by fluorescence-activated cell sorting (FACS), as described above for relative PI fluorescence (FL-2).

\section{Western blot analysis}

Animals were sacrificed by cervical dislocation, and testes were removed. Each tissue sample was homogenized in approximately 20 volumes of buffer using a tissue protein extraction kit (Thermo Scientific) and cleared by centrifugation at $10000 \times \mathrm{g}$ for $5 \mathrm{~min}$ to produce a protein extract. The protein content of the preparation was then determined using a BCA kit (Pierce) with BSA as a standard. Equal concentrations of each sample (50 mg protein) were mixed with the loading buffer, and proteins were then boiled at $100^{\circ} \mathrm{C}$ for $5 \mathrm{~min}$, separated by sodium dodecyl sulfate polyacrylamide gel electrophoresis on $10 \%$ gradient gels, and electrophoretically transferred onto nitrocellulose membranes. For staining, the membranes 
were blocked for $1 \mathrm{~h}$ in TBST with $5 \%$ milk, incubated with a 1:100 dilution of rabbit anti-FasL antibodies (Santa Cruz Biotechnology) at $4{ }^{\circ} \mathrm{C}$ overnight, and subsequently exposed to horseradish peroxidase-conjugated anti-rabbit IgG goat polyclonal antibodies (Santa Cruz Biotechnology). Between each step, the membrane was washed with TBST three times for 10 min each. Bands were visualized with DAB according to the manufacturer's instructions.

\section{Histopathology}

Paraffin-sections were prepared as described above and stained with hematoxylin and eosin according to standard protocols. Sections were then examined and photographed under a Leica microscope.

\section{RESULTS}

\section{Upregulation of FasL in testes after chronic ethanol exposure}

First, we examined the expression of FasL protein in the testes after chronic ethanol exposure. FITC-conjugated flow cytometry analysis showed that after the subtraction of the background control, the percentage of FasL-positive cells was $3.91 \%$ in the testes of saline-fed WT control mice, but was increased to $9.46 \%$ in the testes of ethanol-fed WT mice (Fig. 1A, 1C). Therefore, FasL-positive cells were increased by $5.55 \%$ in mouse testes induced by ethanol. In addition, western blotting analysis revealed that the band of $31 \mathrm{kDa}$ FasL was very weak in the testes of saline-treated mice, whereas a very clear single band of FasL was detected after chronic ethanol exposure (Fig. 2A). These results suggested the expression of FasL protein in the testes was enhanced after chronic ethanol exposure. We also measured the expression in TG mice and observed a higher intensity of FasL expression (Fig. 2B), indicating that FasL was overexpressed in the testes of transgenic mice.

Determination of the relative DNA content of apoptotic nuclei (which were 
hypodiploid due to loss of fragments) by PI staining and cytofluorimetric analysis is a more sensitive way to demonstrate apoptotic cell death (22). As shown in Fig. 3, ethanol-treated WT mice displayed increased apoptotic cells compared with saline-treated WT mice. Transgenic mice also showed slightly more apoptotic cells than their wild-type littermates, which may underlie the slight alterations in testicular morphology in transgenic mice. However, the percentage of apoptotic cells in TG mice (16.22\%) was significantly greater than that in WT mice $(9.46 \%)$ after ethanol exposure. After ethanol injection (1/day), more cells undergoing apoptosis were detected in both WT and TG mice, whereas TG mice showed more significant apoptosis (20) (Fig. 3B). After chronic ethanol exposure, WT mice showed normal testicular morphology (Fig. 3A), whereas TG mice displayed severe injury of spermatogonia and spermatocytes.

\section{DISCUSSION}

Bcl-2 family proteins (23) and p53 (24) are known to be important in the regulation of germ cell apoptosis. The Fas system is also a key regulator of germ cell apoptosis in the testes (10), and upregulation of FasL has been reported in toxicant-induced testicular germ cell apoptosis $(25,26)$. In addition, numerous studies have shown that apoptosis is an important mechanism through which ethanol causes tissue injury $(27,28)$. Recently, Zhu et al showed that apoptosis is an important mechanism through which ethanol causes tissue injury by demonstrating the enhancement of apoptosis by chronic ethanol exposure in testicular germ cells and through measurement of the expression of some apoptosis-related proteins, such as Bcl-2, p53, and FasL (19). While this paper was being written, Eid et al suggested that the Fas system may be involved in germ cell apoptosis in rats treated with chronic ethanol for 12 weeks (29). However, until now, no direct evidence has demonstrated that FasL leads to ethanol-induced 
testicular germ cell apoptosis. In this paper, we showed that induction of testicular germ cell apoptosis after acute ethanol exposure is associated with increased FasL using a transgenic mouse model. Furthermore, we observed severe injury in spermatocytes in some regions and degenerative changes in the epithelial component of the tubules in transgenic mouse testes after acute ethanol exposure. Thus, the background expression of FasL may be directly correlated with the sensitivity of testes to ethanol, indicating that the expression level of FasL could be an important factor for evaluating the risk of tissue damage by ethanol.

This result also could be used to explain (at least in part) the different sensitivities to ethanol-induced tissue damage in human subpopulations. Notably, in certain tissues in FasL-overexpressing transgenic mice, slightly vacuolated spermatogenic epithelium of seminiferous tubules with expanded lumen was observed in the testes using hematoxylin and eosin staining (Fig. 3), consistent with $\mathrm{Hu}$ et al findings (30). We assumed that this damage could have resulted from enhanced apoptosis because of human FasL expression, and this assumption was supported in the present study. PI staining showed that transgenic mice displayed slightly but significantly more apoptotic cells than WT mice (Fig. 1A-D). Moreover, we directly demonstrated that FasL participated in the apoptosis of testicular germ cells after chronic ethanol exposure using FasL-overexpressing transgenic mice.

\section{CONCLUSION}

In the present study, the transgenic mouse model ubiquitously overexpressing human FasL was applied to investigated whether Fas ligand played a role in ethanol-induced testicular germ cell apoptosis. After ethanol injection, WT mice displayed upregulation of Fas ligand in the testes, as demonstrated by fluorescein isothiocyanate-conjugated flow cytometry and western blotting. Moreover, TG mice exhibited significantly more apoptotic germ cells than 
did WT mice after ethanol injection, as shown by DNA fragmentation, propidium iodide staining flow cytometry, and terminal deoxynucleotidyl transferase dUTP nick-end labeling assays. In addition, histopathological examination revealed that degenerative changes in the epithelial component of the tubules occurred in FasL overexpressing transgenic mice, the result suggested that FasL expression determined the sensitivity of the testes to ethanol in mice. In summary, we provided direct evidence demonstrating that Fas ligand mediated apoptosis in testicular germ cells induced by acute ethanol using FasL transgenic mice.

\section{ACKNOWLEDGEMENTS}

This study was supported by the grant from the National Natural Science Foundation of China (81672526).

\section{AUTHORS' NOTE}

Chengdang $\mathrm{Xu}$ and Zhendong Xiang carried out the studies, participated in collecting data, and drafted the manuscript. Denglong Wu and Jingfu Zhang performed the statistical analysis and participated in its design. All authors read and approved the final manuscript. 


\section{REFERENCES}

1. Billig H, Furuta I, Rivier C, Tapanainen J, Parvinen M, Hsueh AJ. Apoptosis in testis germ cells: developmental changes in gonadotropin dependence and localization to selective tubule stages. Endocrinology 1995; 136: 5-12.

2. Guan Y, Liang G, Martin GB, Guan LL. Functional changes in mRNA expression and alternative pre-mRNA splicing associated with the effects of nutrition on apoptosis and spermatogenesis in the adult testis. BMC Genomics 2017; 18: 64.

3. Gao HT, Xu R, Cao WX, Qian LL, Wang M, Lu L et al. Effects of six priority controlled phthalate esters with long-term low-dose integrated exposure on male reproductive toxicity in rats. Food Chem Toxicol 2017:

4. Park JD, Habeebu SS, Klaassen CD. Testicular toxicity of di-(2-ethylhexyl) phthalate in young Sprague-Dawley rats. Toxicology 2002; 171: 105-15.

5. Feng HL, Sandlow JI, Sparks AE, Sandra A, Zheng LJ. Decreased expression of the c-kit receptor is associated with increased apoptosis in subfertile human testes. Fertil Steril 1999; 71: 85-9.

6. Levi M, Popovtzer A, Tzabari M, Mizrachi A, Savion N, Stemmer SM et al. Cetuximab intensifies cisplatin-induced testicular toxicity. Reprod Biomed Online 2016; 33: 102-10.

7. Kyprianou N, Isaacs JT. Activation of programmed cell death in the rat ventral prostate after castration. Endocrinology 1988; 122: 552-62.

8. Atale N, Gupta S, Yadav UC, Rani V. Cell-death assessment by fluorescent and nonfluorescent cytosolic and nuclear staining techniques. J Microsc 2014; 255: 7-19. 
9. Geng YJ. Molecular signal transduction in vascular cell apoptosis. Cell Res 2001; 11: 253-64.

10. Koji T, Hishikawa Y, Ando H, Nakanishi Y, Kobayashi N. Expression of Fas and Fas ligand in normal and ischemia-reperfusion testes: involvement of the Fas system in the induction of germ cell apoptosis in the damaged mouse testis. Biol Reprod 2001; 64: $946-54$.

11. Erkkila K, Kyttanen S, Wikstrom M, Taari K, Hikim AP, Swerdloff RS et al. Regulation of human male germ cell death by modulators of ATP production. Am J Physiol Endocrinol Metab 2006; 290: E1145-54.

12. Nagata S, Golstein P. The Fas death factor. Science 1995; 267: 1449-56.

13. Fan XQ, Guo YJ. Apoptosis in oncology. Cell Res 2001; 11: 1-7.

14. Oh SI, Lee MS, Kim CI, Song KY, Park SC. Aspartate modulates the ethanol-induced oxidative stress and glutathione utilizing enzymes in rat testes. Exp Mol Med 2002; 34: 47-52.

15. Rosenblum E, Gavaler JS, Van Thiel DH. Lipid peroxidation: a mechanism for ethanol-associated testicular injury in rats. Endocrinology 1985; 116: 311-8.

16. Saito K, Niijima A, Kamite E, Watanabe M. Bisphenol A and estrone-induced developmental effects in early chick embryos. Environ Toxicol 2012; 27: 58-62.

17. Woolveridge I, de Boer-Brouwer M, Taylor MF, Teerds KJ, Wu FC, Morris ID. Apoptosis in the rat spermatogenic epithelium following androgen withdrawal: changes in apoptosis-related genes. Biol Reprod 1999; 60: 461-70.

18. Zhu Q, Van Thiel DH, Gavaler JS. Effects of ethanol on rat Sertoli cell function: 
studies in vitro and in vivo. Alcohol Clin Exp Res 1997; 21: 1409-17.

19. Zhu Q, Meisinger J, Emanuele NV, Emanuele MA, LaPaglia N, Van Thiel DH. Ethanol exposure enhances apoptosis within the testes. Alcohol Clin Exp Res 2000; 24: $1550-6$.

20. Jankala H, Eriksson PC, Eklund K, Sarviharju M, Harkonen M, Maki T. Effect of chronic ethanol ingestion and gender on heart left ventricular p53 gene expression. Alcohol Clin Exp Res 2005; 29: 1368-73.

21. Herrero R, Kajikawa O, Matute-Bello G, Wang Y, Hagimoto N, Mongovin S et al. The biological activity of FasL in human and mouse lungs is determined by the structure of its stalk region. J Clin Invest 2011; 121: 1174-90.

22. Nicoletti I, Migliorati G, Pagliacci MC, Grignani F, Riccardi C. A rapid and simple method for measuring thymocyte apoptosis by propidium iodide staining and flow cytometry. J Immunol Methods 1991; 139: 271-9.

23. Kuerban M, Naito M, Hirai S, Terayama H, Qu N, Musha M et al. Involvement of Fas/Fas-L and Bax/Bcl-2 systems in germ cell death following immunization with syngeneic testicular germ cells in mice. J Androl 2012; 33: 824-31.

24. Beumer TL, Roepers-Gajadien HL, Gademan IS, van Buul PP, Gil-Gomez G, Rutgers DH et al. The role of the tumor suppressor p53 in spermatogenesis. Cell Death Differ 1998; 5: 669-77.

25. Banerjee B, Chakraborty S, Ghosh D, Raha S, Sen PC, Jana K. Benzo(a)pyrene Induced p53 Mediated Male Germ Cell Apoptosis: Synergistic Protective Effects of Curcumin and Resveratrol. Front Pharmacol 2016; 7: 245. 
26. Richburg JH, Nanez A, Williams LR, Embree ME, Boekelheide K. Sensitivity of testicular germ cells to toxicant-induced apoptosis in gld mice that express a nonfunctional form of Fas ligand. Endocrinol 2000; 141: 787-93.

27. Luo XJ, Liu B, Dai Z, Li TB, Li NS, Zhang XJ et al. Expression of apoptosis-associated microRNAs in ethanol-induced acute gastric mucosal injury via JNK pathway. Alcohol 2013; 47: 481-93.

28. Singhal PC, Reddy K, Ding G, Kapasi A, Franki N, Ranjan R et al. Ethanol-induced macrophage apoptosis: the role of TGF-beta. J Immunol 1999; 162: 3031-6.

29. Eid NA, Shibata MA, Ito Y, Kusakabe K, Hammad H, Otsuki Y. Involvement of Fas system and active caspases in apoptotic signalling in testicular germ cells of ethanol-treated rats. Int J Androl 2002; 25: 159-67.

30. Hu JH, Jiang J, Ma YH, Yang N, Zhang MH, Wu M et al. Enhancement of germ cell apoptosis induced by ethanol in transgenic mice overexpressing Fas Ligand. Cell Res 2003; 13: 361-7. 
Table 1: Germ cells apoptosis between the WT and transgenetic mice

\section{Control Treatment (12 weeks)}

$\begin{array}{lll}\text { WT } & 3.91 \% & 9.46 \% \\ \text { TG } & 6.90 \% & 16.22 \%\end{array}$



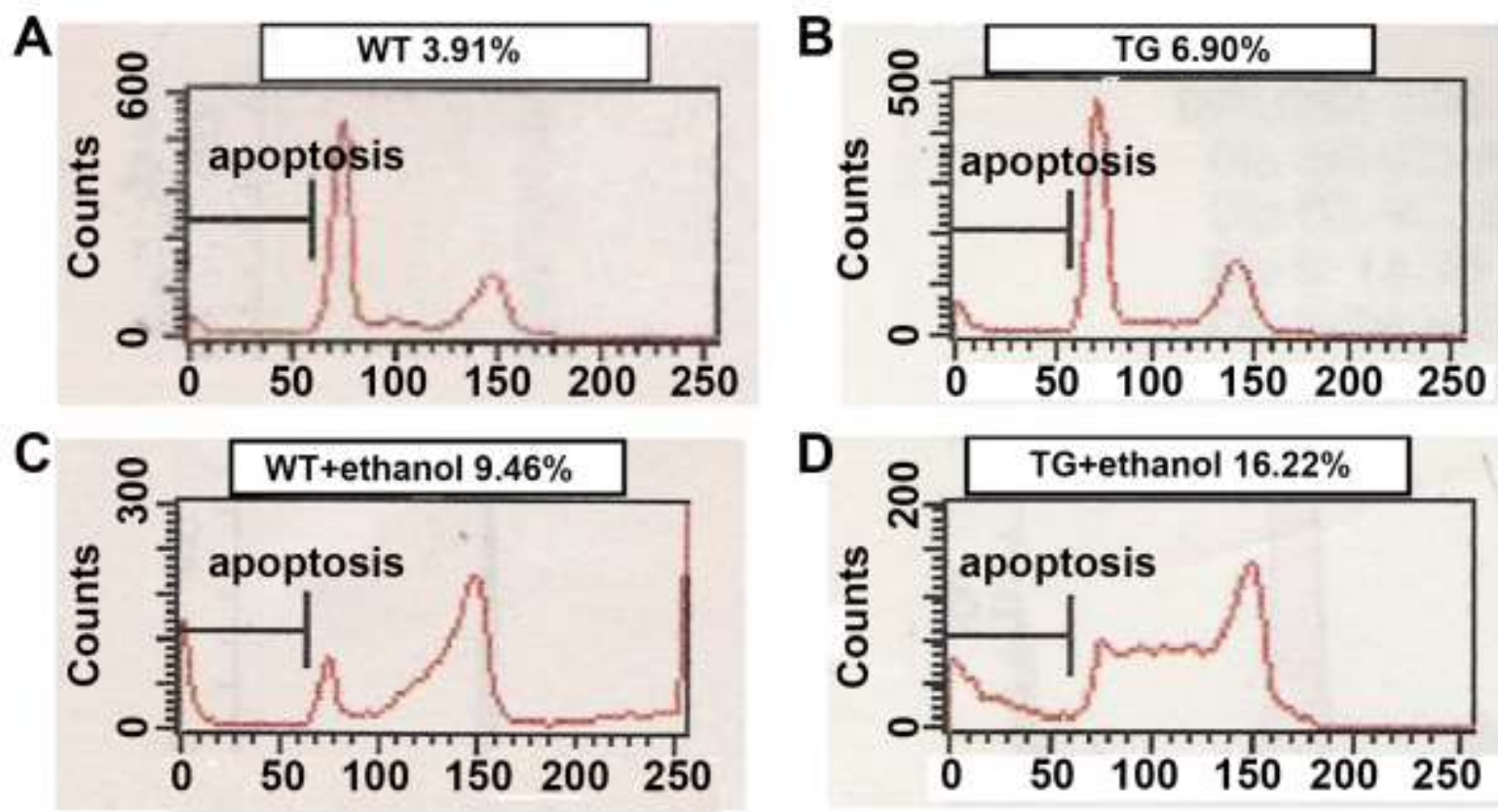

Fig. 1: Flow cytometry analysis of apoptosis in the testes of wild-type and transgenic mice after ethanol feeding for 12 weeks by PI staining. The percentages of apoptotic cells in all testes cells are indicated by the cursor. $\mathrm{n}=3$ mice were individually analyzed in two separate experiments for each group $(p<0.05)$.

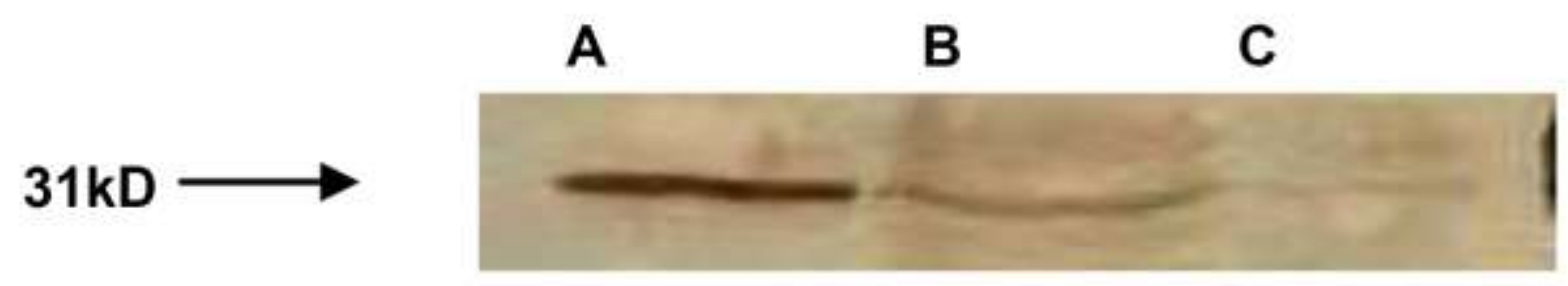

Fig. 2: The expression of FasL protein in the testes after ethanol feeding. Western blot analysis showed that FasL protein was increased in the testes after ethanol feeding. Lane A: wild-type mice after ethanol feeding, lane $\mathrm{B}$ : transgenic mice, lane $\mathrm{C}$ : wild-type mice with saline injection as a control. $\mathrm{n}=3$ for each group. 

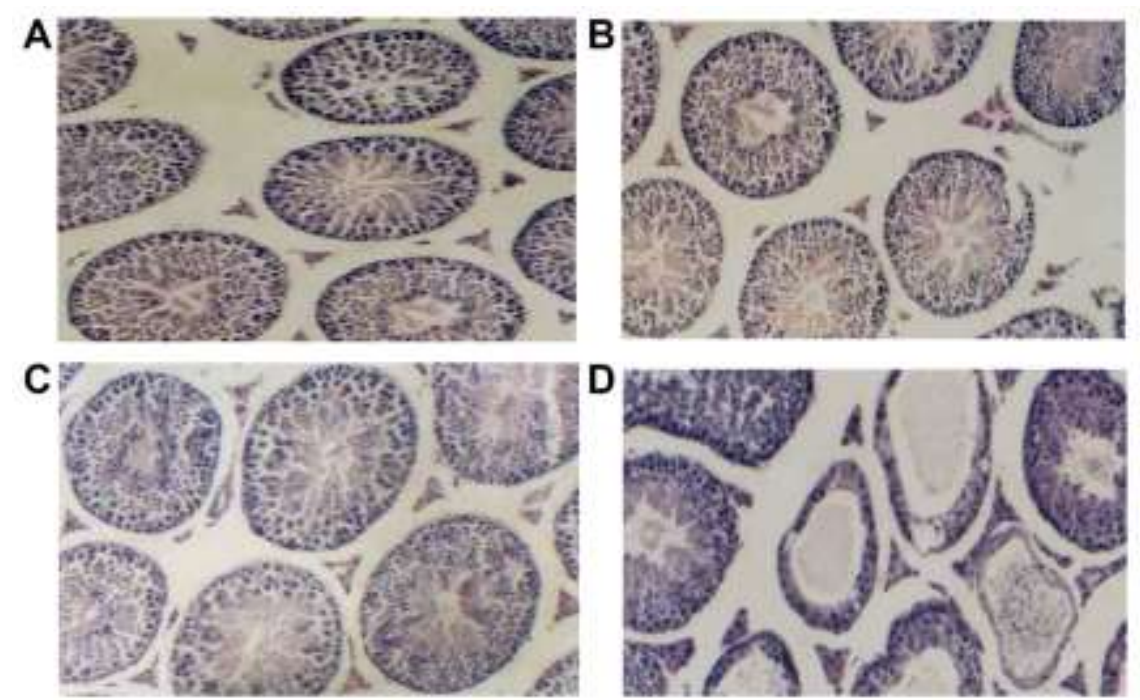

Fig. 3: Testicular histopathology induced by ethanol. Note expanded lumen in TG mice and degenerative changes in the epithelial component of the tubules in TG mice compared with wild-type mice after ethanol exposure. (C): WT+ethanol, (D): TG+ethanol. $\mathrm{n}=5$ in each group.

\section{Affiliations:}

${ }^{1}$ Department of Urology, Tongji Hospital, Tongji University School of Medicine, 389 Xincun Road, Shanghai, 200065 China.

${ }^{2}$ Department of Urology, Tongren Hospital, Shanghai Jiaotong University School of Medicine, Shanghai, China.

\section{Correspondence:}

Dr W Le

Department of Urology

Tongji Hospital, Tongji University School of Medicine

389 Xincun Road

Shanghai

China

Fax: +86-021-64085875

E-mail: lewei0411@163.com

Short title: Germ Cell Apoptosis Induced by Chronic Ethanol

Synopsis: The present study mainly carried on chronic effects on FASL transgenic mice. In 
this study, TUNEL, flow cytometry and western blot techniques were applied directly to investigate the influence of ethanol on mammalian animal testis. After treatment by ethanol, there exists irreversible apoptosis on germline cells in testis especially in transgenic mice testis. There is no doubt that the present study provides the vigorous evidence of chronic influence on germline cells. 